\title{
Phase and intensity dynamics of a two-frequency laser submitted to resonant frequency-shifted feedback
}

\author{
J. Thévenin, ${ }^{1}$ M. Romanelli, ${ }^{1}$ M. Vallet, ${ }^{1}$ M. Brunel, ${ }^{1}$ and T. Erneux ${ }^{2}$ \\ ${ }^{1}$ Institut de Physique de Rennes, Université Rennes I - CNRS UMR 6251, Campus de Beaulieu, 35042 Rennes Cedex, France \\ ${ }^{2}$ Université Libre de Bruxelles, Optique Non Linéaire Théorique, Campus Plaine, CP 231, 1050 Bruxelles, Belgium
}

(Received 13 June 2012; published 11 September 2012)

\begin{abstract}
We propose an experimental and theoretical study of the dynamical regimes observed when the two modes of a two-frequency solid-state laser are coupled by frequency-shifted optical feedback. The detuning associated to the feedback is close to the frequency of the laser relaxation oscillations. Special attention is devoted to the dynamics of the phase of the beat note between the modes relative to the phase of an external radio-frequency reference. In particular, we analyze in detail the transition from phase locking to the external reference to phase drift. This transition occurs through a window of frequency locking without phase locking [Thévenin, Romanelli, Vallet, Brunel, and Erneux, Phys. Rev. Lett. 107, 104101 (2011)]. Furthermore, a large variety of dynamical behaviors, such as weak intensity modulation, self-pulsing, and chaos, are experimentally found. These results are successfully reproduced by a theoretical model based on coupled rate equations. The extension of the model to the description of other active media such as semiconductor lasers is briefly outlined.
\end{abstract}

DOI: 10.1103/PhysRevA.86.033815

PACS number(s): 42.65.Sf, 42.55.Xi, 05.45.Xt

\section{INTRODUCTION}

It is well known that lasers are extremely sensitive to optical feedback [1]. This is often seen as a problem to control, but optical feedback can also be used to stabilize the laser intensity and frequency [2], to produce pulsed operation [3], and to detect very weak amounts of light [4]. In particular, class-B lasers are extremely sensitive to frequency-shifted feedback (FSF) when the frequency shift is close to the laser-relaxationoscillation (RO) frequency $f_{R}$. This fact has been exploited, for instance, for imaging purposes [5].

FSF was recently used to lock the beat note between the polarization modes of a two-frequency laser against a microwave synthesizer [6]. This technique allows one to obtain a radio-frequency (RF) clock signal on an optical carrier or to ensure pulse-to-pulse phase coherence for Lidar-Radar distance and velocity measurements [7]. The locking properties of the two-frequency laser under FSF were described by an Adler-type equation, governing the relative phase $\Phi$ between the laser beat note and the reference signal [8]. If the frequency difference $\Delta v$ between the two oscillators is lower (higher) than the frequency detuning $f_{A}$, determined by the amount of fed-back light, the two oscillators are locked (unlocked). Recently, we have shown that such a system can exhibit richer synchronization properties. Specifically, we have found experimental and numerical evidence of a regime of frequency locking without phase locking [9]. This regime, also called the phase entrainment [10,11], phase trapping [12], or bounded phase $[13,14]$ in previous literature, appears when $\Delta v$ is bigger than $f_{A}$, thus extending the synchronization region beyond $f_{A}$. In such a case, the amplitudes of the coupled oscillators can no longer be considered nearly constant and play an important role in the dynamics.

The aim of the present paper is to give a comprehensive experimental and theoretical study of the different dynamical regimes of a two-frequency laser submitted to resonant FSF. In Sec. II, we describe the experimental setup and the associated rate-equation model. Section III is devoted to a global presentation of the dynamics of our system as predicted by the model. To this end, we numerically compute intensity and phase bifurcation diagrams using $\Delta v$ as a control parameter. This study permits us to point out the occurrence of several different regimes. Section IV compares systematically the experimental results with the theoretical predictions. Finally, our main results are summarized and discussed in Sec. V.

\section{SYSTEM DESCRIPTION}

\section{A. Experimental setup}

We consider a two-frequency Nd:YAG diode-pumped solid-state laser [15], followed by the feedback cavity, as shown in Fig. 1(a) [8]. The laser cavity of length $L=75 \mathrm{~mm}$ is closed on one side by a high-reflection plane mirror, which is coated on the 5-mm-long Nd:YAG active medium, and on the other side by a concave mirror (with a radius of curvature of $100 \mathrm{~mm}$ and an intensity transmission of $1 \%$ at the lasing wavelength $\lambda=1064 \mathrm{~nm}$ ). The active medium is pumped by a laser diode emitting at $808 \mathrm{~nm}$. A 1-mm-thick silica étalon ensures single longitudinal-mode oscillation. Two eigenmodes $E_{x}$ and $E_{y}$, polarized along $\hat{x}$ and $\hat{y}$ with eigenfrequencies $v_{x}$ and $v_{y}$, respectively, oscillate simultaneously. An intracavity birefringent element (here two rotated quarter-wave plates) induces a frequency difference $\Delta v_{0}=v_{y}-v_{x}$ that is finely tunable from 0 to $1 \mathrm{GHz}$ (half the free spectral range of the laser cavity). In our experiments, we choose $\Delta v_{0}=200 \mathrm{MHz}$ in order to avoid coherent effects in the cross saturation of the active medium; under this condition, we can assume that each laser field interacts with its own population-inversion reservoir [16]. It is important to note that $\Delta v_{0}$ is much more stable than $v_{x}$ and $v_{y}$ since the two eigenmodes share the same cavity. This implies that frequency fluctuations due to technical noise cancel out almost perfectly on the frequency difference [15]. The typical power emitted by the two-frequency laser is $10 \mathrm{~mW}$ when pumped with $500 \mathrm{~mW}$. The feedback cavity contains a Bragg cell, driven by a stable RF synthesizer, which provides an external phase reference. Next, a quarter-wave plate at $45^{\circ}$ followed by a mirror flips the $x$ and $y$ polarizations, 

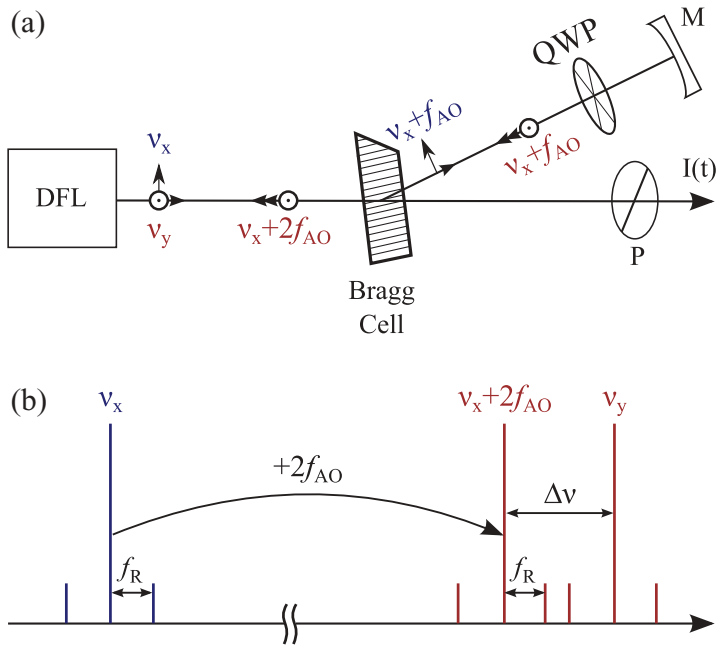

FIG. 1. (Color online) (a) Experimental setup. DFL: dualfrequency laser. QWP: quarter-wave plate. M: mirror. P: polarizer. (b) Diagram of the main frequencies involved in the dynamics of the system. $v_{x}$ corresponds to a wave polarized along $x$ while $v_{y}$ and $v_{x}+2 f_{A O}$ correspond to $y$ polarization.

and finally, the laser beam is reinjected in the laser cavity after again crossing the Bragg cell. As a result, an $x$-polarized field oscillating at the frequency $v_{y}+2 f_{A O}$ and a $y$-polarized field oscillating at the frequency $v_{x}+2 f_{A O}$ are reinjected in the laser. We choose $f_{A O}$ around $100 \mathrm{MHz}$ so that the frequency detuning $\Delta v=\Delta v_{0}-2 f_{A O}$ is on the order of the RO frequency $f_{R}$ as shown in Fig. 1(b). We note that the optical reinjection has no direct effect on $E_{x}$ because the frequency difference between $v_{x}$ and $v_{y}+2 f_{A O}$ is too large. For the same reason, multiple round trips in the feedback cavity have no effect on the dynamics. Finally, we detect the laser output with a fast photodiode (3-GHz analogic bandwidth) after a crossed polarizer, thus obtaining an electrical signal proportional to $I=\left|E_{x}+E_{y}\right|^{2}$. The signal is then analyzed with an electrical spectrum analyzer and a digital oscilloscope (40 GS/s, analogic bandwidth $6 \mathrm{GHz}$ ).

\section{B. Theoretical model}

We start from the Lang-Kobayashi two-mode rate equations already introduced in Ref. [8]:

$$
\begin{aligned}
\frac{d E_{x}}{d t}= & {\left[-\Gamma_{x}+\kappa\left(n_{x}+\beta n_{y}\right)\right] \frac{E_{x}}{2}+2 i \pi v_{x} E_{x}+\tilde{E}_{x} } \\
\frac{d E_{y}}{d t}= & {\left[-\Gamma_{y}+\kappa\left(n_{y}+\beta n_{x}\right)\right] \frac{E_{y}}{2}+2 i \pi v_{y} E_{y} } \\
& +\tilde{E}_{y}+\gamma_{e} E_{x}(t-\tau) e^{4 i \pi f_{A O} t+i \Psi} \\
\frac{d n_{x, y}}{d t}= & \gamma_{\| \mid} P_{x, y}-\left[\gamma_{\|}+\zeta\left(\left|E_{x, y}\right|^{2}+\beta\left|E_{y, x}\right|^{2}\right)\right] n_{x, y}+\tilde{n}_{x, y} .
\end{aligned}
$$

In these equations, $n_{x, y}$ are the two population inversions; $\gamma_{\|}$is the population-inversion lifetime; $P_{x, y}$ are the pumping rates; $\kappa$ and $\zeta$ are the atom-field coupling coefficients, respectively; $\beta$ accounts for the cross saturation in the active medium; $\tilde{E}_{x, y}$ and $\tilde{n}_{x, y}$ are noise terms representing spontaneous emission; $\Gamma_{x, y}$ are the cavity lifetimes, such that $\Gamma_{x, y}=-c / 2 L \ln \left[R_{1} R_{2}(1-\right.$ $\left.\left.\delta_{x, y}\right)^{2}\right]$, with $L$ being the laser-cavity length and $R_{1}$ and $R_{2}$ being the reflectivities of the laser mirrors, respectively; $\delta_{x, y}$ are the single-pass loss coefficients; $\gamma_{e}$ is the feedback strength [4] and accounts for the reflectivity of the mirror $M$, the diffraction efficiency, the transmission of all the various optical components, and the mode matching between the feedback beam and the laser intracavity field; and $\tau$ and $\Psi$ are, respectively, the delay and the phase associated to the feedback optical path.

Equations (1)-(3) can be simplified by assuming identical losses and pump rates, i.e., $\Gamma_{x}=\Gamma_{y}=\gamma$ and $P_{x}=P_{y}=P$. In addition, we neglect the noise terms. Numerical simulations indicate that they do not play a significant role in the dynamics. Under our experimental conditions, the feedback is seen as instantaneous by the laser. Its dynamics evolves on a time scale $\sim 1 / f_{R}=14 \mu \mathrm{s}$, which is much slower than $\tau=5.1 \mathrm{~ns}$. This motivates neglecting $\tau$ in Eqs. (1)-(3) without altering the numerical solutions. This assumption has been checked numerically in a few typical cases. We introduce the following scaled variables:

$$
\begin{aligned}
E_{x} & =\bar{E}_{x} \sqrt{\frac{\gamma_{\|}}{\zeta}} e^{2 i \pi v_{x} t} e^{-i \psi}, \\
E_{y} & =\bar{E}_{y} \sqrt{\frac{\gamma_{\|}}{\zeta}} e^{2 i \pi\left(v_{x}+2 f_{A O}\right) t}, \\
n_{x, y} & =\frac{\gamma}{\kappa} N_{x, y}, \\
\Delta & =\frac{v_{y}-v_{x}-2 f_{A O}}{\gamma},
\end{aligned}
$$

and decompose the fields into amplitude and phase $\bar{E}_{x, y}=$ $\sqrt{I_{x, y}} e^{i \phi_{x, y}}$. Since $\phi_{x}$ is constant, the equations reduce to

$$
\begin{aligned}
\frac{1}{\gamma} \frac{d I_{x}}{d t} & =\left[N_{x}+\beta N_{y}-1\right] I_{x}, \\
\frac{1}{\gamma} \frac{d I_{y}}{d t} & =\left[N_{y}+\beta N_{x}-1\right] I_{y}+4 \pi K \sqrt{I_{x} I_{y}} \cos \Phi, \\
\frac{1}{\gamma} \frac{1}{2 \pi} \frac{d \Phi}{d t} & =\Delta-K \sqrt{\frac{I_{x}}{I_{y}}} \sin \Phi, \\
\frac{1}{\gamma} \frac{d N_{x, y}}{d t} & =\epsilon\left[r-\left(1+I_{x, y}+\beta I_{y, x}\right) N_{x, y}\right],
\end{aligned}
$$

where we have introduced the normalized feedback strength $K=\frac{\gamma_{e}}{2 \pi \gamma}=\frac{f_{A}}{\gamma}$ (where $f_{A}$ is the Adler frequency), the normalized pump rate $r=\frac{k P}{\gamma}$, and the small parameter $\epsilon=\frac{\gamma_{\|}}{\gamma}$. Note that $\Phi=\phi_{y}-\phi_{x}$ is the phase of the slowly varying amplitude $\bar{E}_{y}$ relative to the phase $2 \pi\left(v_{x}+2 f_{A O}\right) t$ of the reinjected field; alternatively, $\Phi$ can also be seen as the phase between the beat note oscillating at $\Delta v_{0}$ and the reference oscillating at $2 f_{A O}$. Equations (8)-(11) are appropriate for comparative studies between theory and experiments since we have experimental access to the intensities and to the relative phase. For all our numerical simulations, we consider, however, the complex fields. The reasons are twofold. First, the $\sqrt{I_{y}}$ term in the denominator of Eq. (10) causes trouble when $I_{y}$ is small; second, Eqs. (8)-(11) possess a singular limit, i.e., they have a nonphysical solution when $\epsilon \rightarrow 0$. Both problems can be removed working with properly scaled 
TABLE I. Numerical values of the parameters used for the simulations.

\begin{tabular}{lcc}
\hline \hline Parameter & & Value \\
\hline Population-inversion lifetime & $\left(1 / \gamma_{\|}\right)$ & $230 \mu \mathrm{s}$ \\
Photon cavity lifetime & $(1 / \gamma)$ & $4.32 \mathrm{~ns}$ \\
RO angular frequency & $\left(\Omega_{R}\right)$ & $4.49 \times 10^{5} \mathrm{rad} \mathrm{s}^{-1}$ \\
Cross-saturation coupling & $(\beta)$ & 0.6 \\
Pump rate & $(\eta)$ & 1.2 \\
Feedback strength & $\left(\gamma_{e}\right)$ & $3.59 \times 10^{5} \mathrm{rad} \mathrm{s}^{-1}$ \\
\hline \hline
\end{tabular}

complex fields and using the adimensional time $s=\Omega_{R} t$, where $\Omega_{R}=\sqrt{\gamma_{\|} \gamma[r(1+\beta)-1]}$ is the RO angular frequency [17]. Equations (1)-(3) are then rewritten in a more convenient way for numerical integration:

$$
\begin{aligned}
\frac{d e_{x}}{d s}= & \frac{\left(m_{x}+\beta m_{y}\right)}{1+\beta} \frac{e_{x}}{2}, \\
\frac{d e_{y}}{d s}= & \frac{\left(m_{y}+\beta m_{x}\right)}{1+\beta} \frac{e_{y}}{2}+i \Delta \Omega e_{y}+\frac{\gamma_{e}}{\Omega_{R}} e_{x}, \\
\frac{d m_{x, y}}{d s}= & 1-\left(\left|e_{x, y}\right|^{2}+\beta\left|e_{y, x}\right|^{2}\right) \\
& -\epsilon^{\prime} m_{x, y}\left[1+(\eta-1)\left(\left|e_{x, y}\right|^{2}+\beta\left|e_{y, x}\right|^{2}\right)\right],
\end{aligned}
$$

where $e_{x, y}=\bar{E}_{x, y} \sqrt{\gamma_{\|} \gamma} / \Omega_{R}, N_{x, y}=\frac{1}{1+\beta}\left(1+\frac{\Omega_{R}}{\gamma} m_{x, y}\right), \eta=$ $r(1+\beta), \epsilon^{\prime}=\gamma_{\|} / \Omega_{R}$, and $\Delta \Omega=2 \pi\left(v_{y}-v_{x}-2 f_{A O}\right) / \Omega_{R}$.

From these equations, time series and spectra are calculated using a fourth-order Runge-Kutta algorithm with an adaptive time step. The spectra are obtained by convoluting the Fourier transform of the time series with a gate function that reproduces the spectral resolution of the electrical spectrum analyzer, which can vary from $1 \mathrm{~Hz}$ to $500 \mathrm{kHz}$ depending on the frequency span. Table I lists the values of the parameters used in the simulations. All of these parameters are experimentally measured. The cross-saturation coupling $\beta$ is measured using the technique discussed in Ref. [18] while the other parameters are deduced from Ref. [19] and the references therein.

\section{BIFURCATION DIAGRAMS}

In this section we propose a general overview of the dynamics of the system. To this end, we calculate numerically bifurcation diagrams for the intensity and phase as a function of the control parameter $\Delta v$. The bifurcation diagrams allow us to clearly identify several distinct regimes, which are compared to the experimental ones in the following section.

The intensity bifurcation diagrams, shown in Fig. 2, are obtained in the following way. For a given value of $\Delta v$, a time series of $I_{x}, I_{y}$, and $I_{x y}=\left|\bar{E}_{x}+\bar{E}_{y}\right|^{2}$ is calculated by numerical integration of Eqs. (12)-(14). The extrema of the long-time solutions are extracted and represented as functions of $\Delta v$. Taking $\bar{E}_{x, y}$ rather than $E_{x, y}$ allows us to remove the fast $200-\mathrm{MHz}$ oscillation from the time series (see also Figs. 4-9). $\Delta v$ is progressively increased, and the previous numerical long-time solution serves as an initial condition for the following computation. Figure 2 describes the behavior of the $y$ eigenstate [Fig. 2(a)], of the $x$ eigenstate [Fig. 2(b)], and of the beating [Fig. 2(c)]. Starting from high values of
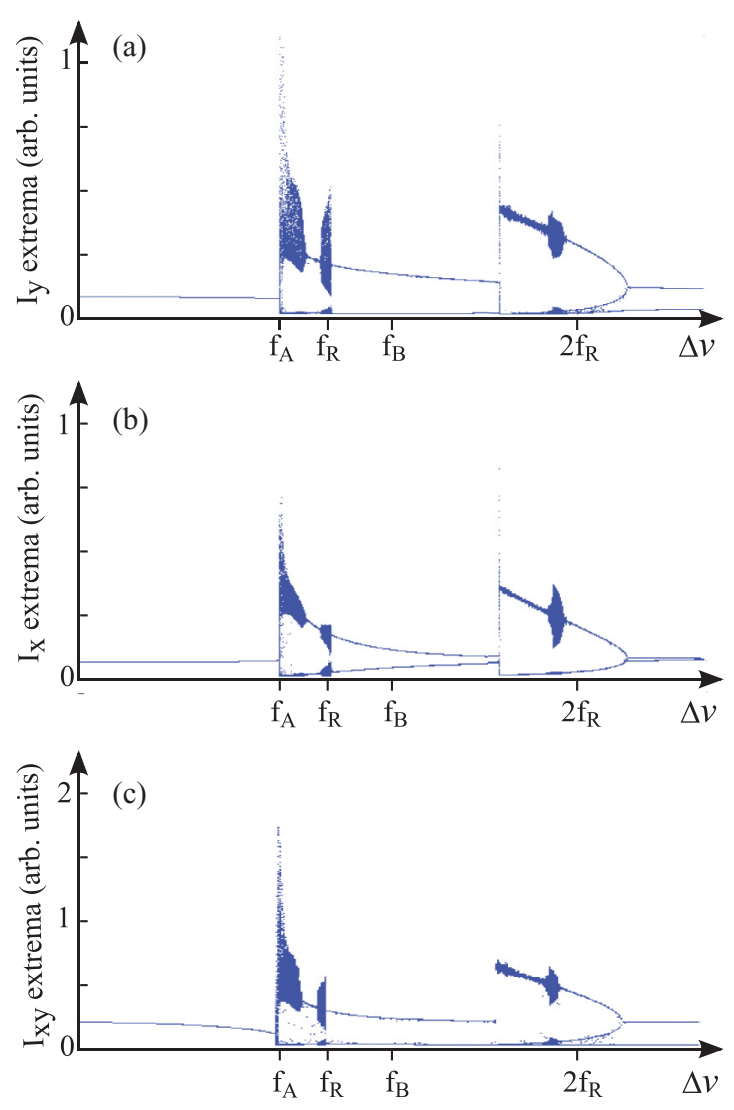

FIG. 2. (Color online) Computed bifurcation diagrams as $\Delta v$ is varied of (a) $I_{y}=\left|\bar{E}_{y}\right|^{2}$, (b) $I_{x}=\left|\bar{E}_{x}\right|^{2}$, and (c) $I_{x y}=\left|\bar{E}_{x}+\bar{E}_{y}\right|^{2}$. Note that the vertical scales are different.

$\Delta v$, we see that when $\Delta v$ is larger than $2 f_{R}$, the intensity of the $y$ eigenstate is slowly modulated by the feedback at a frequency $\Delta v$. This modulation results in two extrema lines in Fig. 2(a). We stress that, even in absence of coherent optical feedback, the $x$ eigenstate is in turn modulated (the amplitude of the modulation being about ten times smaller). This is due to the coupling in the active medium, accounted for by the $\beta$ parameter, implying that our system is substantially different from a master-slave configuration. When $\Delta v$ approaches $2 f_{R}$, a period-doubling bifurcation appears. The amplitude of this subharmonic modulation increases as $\Delta v$ decreases and suddenly jumps to a simple harmonic modulation. Close to resonance, i.e., when $\Delta v \simeq f_{R}$, the two eigenstates are more sensitive to perturbations, and the modulation develops gradually into a pulsed regime with a $\Delta v$ repetition rate (see Fig. 7). For $f_{A}<\Delta v<f_{R}$, the intensity dynamics becomes more complex (see Fig. 8). The power spectrum spans continuously a broad frequency range, much larger than the characteristic frequencies $f_{R}$ and $f_{A}$ and suggests that this regime is chaotic. Finally, when $\Delta v<f_{A}$, the beating of the two eigenstates is phase locked to the external reference, and as a result, the beat-note signal consists of a stable oscillation of constant amplitude.

For the phase, we obtain the bifurcation diagram by simulating Eqs. (12)-(14) and then by determining $\Phi$. The local extrema $X_{\Phi}$ of $\Phi$ are plotted as a function of $\Delta v$ (Fig. 3). Before discussing the diagram, we recall for the sake of comparison the behavior that is expected for the standard 


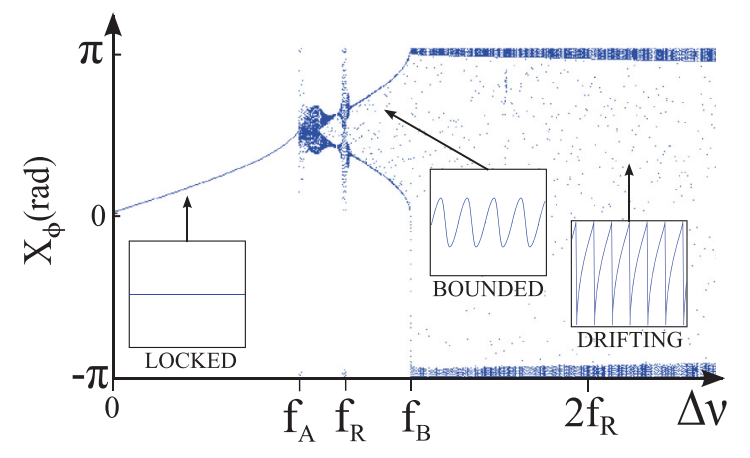

FIG. 3. (Color online) Computed bifurcation diagram for the relative phase $\Phi$ as a function of $\Delta v$. Three qualitatively different behaviors appear: (a) $\Delta v<f_{A}$ (phase locking), (b) $f_{A}<\Delta v<f_{B}$ (frequency locking without phase locking), and (c) $\Delta v>f_{B}$, (phase drift, corresponding to the fact that the frequencies are unlocked). Insets: Time series of $\Phi(t)$.

Adler equation, which is obtained from Eq. (10) if the intensity dynamics is discarded by setting $I_{x}=I_{y}$ [8]. Such an equation reads

$$
\frac{\dot{\Phi}}{2 \pi}=\Delta v-f_{A} \sin \Phi .
$$

If $\left|\Delta v / f_{A}\right| \leqslant 1$, a stable stationary solution exists, and the two oscillators are phase locked. On the other hand, if $\left|\Delta v / f_{A}\right|>1$, the two oscillators cannot synchronize, and their relative phase drifts indefinitely in time.

From the bifurcation diagram, we note that the standard Adler equation captures well the dynamics when $\Delta v \in\left[0 ; f_{A}\right]$. In this range, $\Phi$ is constant over time; therefore, the two oscillators are phase locked. In contrast with the standard behavior described by Eq. (15), however, in our model the relative phase remains bounded beyond $f_{A}$ up to a certain value $f_{B}$ of $\Delta v$ (except for two narrow regions close to $\Delta v=f_{R}$ and $\Delta v=f_{A}$ ). This implies that the average frequency of the beat note coincides with the reference frequency; in other words, the interval $f_{A}<\Delta v<f_{B}$ defines a region of frequency locking without phase locking. The oscillators unlock completely only when $\Delta v>f_{B}$. Since $\Phi(t)$ is numerically restricted to the interval $[-\pi ; \pi]$, an unbounded, drifting phase results in a "sawtoothlike" time series (see the insets in Fig. 3) with abrupt vertical jumps as $\Phi(t)$ reaches the value of $\pi$. As a consequence, the values $-\pi$ and $\pi$ appear as local extrema in the time series of an unbounded-phase regime.

We stress that, even if the intensity and phase dynamics are coupled, the sequence of bifurcations is not the same. Indeed, while $f_{A}$ is a bifurcation point for both intensity and phase, at $f_{B}$ where the phase becomes unbounded, no bifurcation occurs for the intensity. We comment further on this point later. Furthermore, the period-doubling bifurcation on the intensity around $2 f_{R}$ produces no special signature on the phase dynamics because phase unbounding has already occurred.

\section{COMPARISON BETWEEN EXPERIMENTAL AND THEORETICAL RESULTS}

In this section, we consider in detail each dynamical regime and systematically compare the experimental data
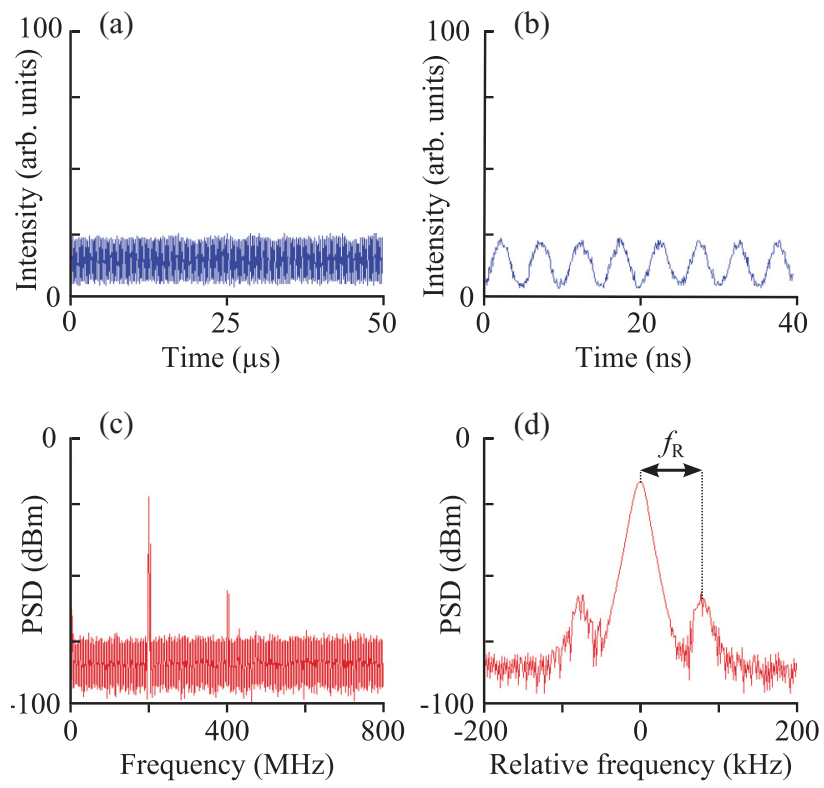

FIG. 4. (Color online) $\Delta v<f_{A}$. (a), (b) Experimental time series of $I=\left|E_{x}+E_{y}\right|^{2}$. (c), (d) Corresponding experimental power spectral density.

with the simulations of the theoretical model. We begin our analysis by discussing the simplest and most typical situations, i.e., the phase-locking regime for small $\Delta v$ and the phase-drifting regime for large $\Delta v$. Then, we discuss the different time-dependent regimes that take place between these two extreme cases. Experimentally, we control $\Delta v$ by changing the frequency $f_{A O}$ of the RF synthesizer.

\section{A. Intensity measurements}

\section{Phase locking}

If $\Delta v<f_{A}$, the laser beat-note frequency $\Delta v_{0}=v_{y}-v_{x}$ locks on the RF drive frequency $2 f_{A O}$. The intensity $I=$ $\left|E_{x}+E_{y}\right|^{2}$ exhibits a pure sinusoidal oscillation [Figs. 4(a) and 4(b)], whose power spectrum consists mainly of a peak at $2 f_{A O}$. The residual peak at $400 \mathrm{MHz}$ in Fig. 4(c) is attributed to the nonresonant feedback of the $x$ eigenstate into the $y$ eigenstate. In Fig. 4(d) the RO peak, whose intensity is $-40 \mathrm{~dB}$ relative to the main peak, is also visible. The linewidth of the beat note could not be resolved with the electrical spectrum analyzer; all that can be said is that it is smaller than $1 \mathrm{~Hz}$. This tends to demonstrate that the RF-synthesizer stability was successfully transferred to the optical beat note. These results are in agreement with the numerical simulations.

\section{Phase drifting: Modulated beat note}

When $\Delta v$ is significantly larger than $f_{A}$ (typically $\Delta v \geqslant$ $3 f_{A}$ ), the oscillators keep their own frequencies when they are coupled. As a result, the detected intensity displays an amplitude modulation at the frequency $\Delta v$, resulting from the beating between $\Delta v_{0}$ and $2 f_{A O}$ [Figs. 5(a) and 5(b)]. The measured and simulated power spectra, shown in Figs. 5(c) and $5(\mathrm{~d})$, respectively, consist of a peak at $2 f_{A O}\left(2 f_{A O}\right.$ is taken as the frequency zero for clarity), two peaks at $2 f_{A O} \pm \Delta v$, and the $\mathrm{RO}$ peaks. 

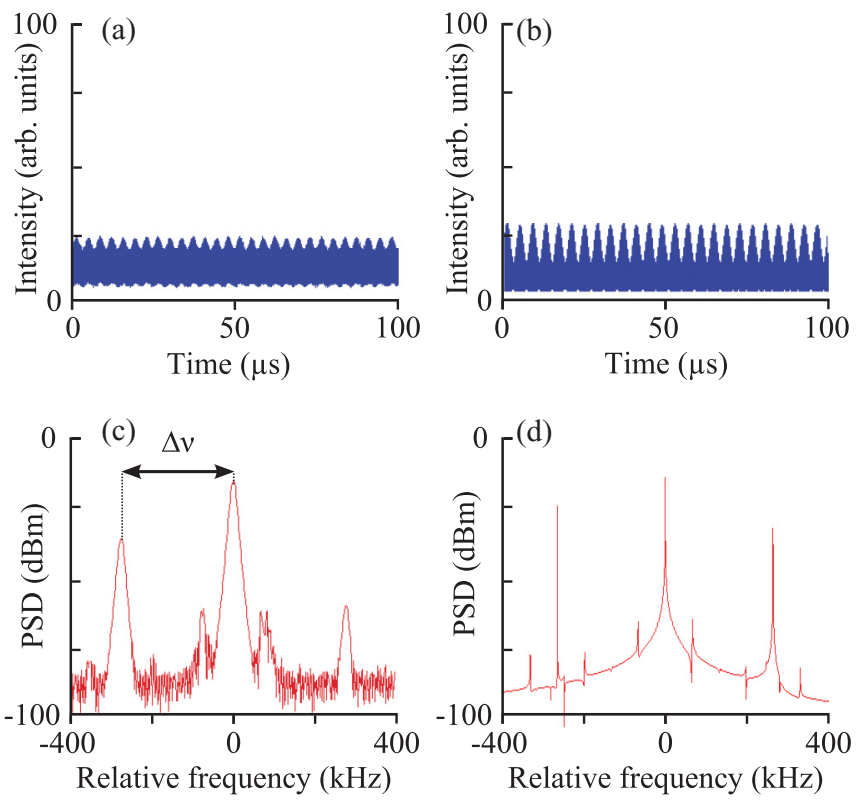

FIG. 5. (Color online) $\Delta v=3 f_{A}$. (a) Experimental and (b) simulated time series of $I=\left|E_{x}+E_{y}\right|^{2}$. (c) Experimental and (d) simulated power spectral density. The displayed frequencies are relative to $2 f_{A O}$.

\section{Period doubling $\left(\Delta v \approx 2 f_{R}\right)$}

When $\Delta v$ is reduced starting from the previous case, a period-doubling bifurcation appears as shown by the experimental and simulated time series [Figs. 6(a) and 6(b)]. The associated power spectra [Figs. 6(c) and 6(d)] allow us to understand that this bifurcation arises when the RO peak of one mode starts to overlap with the RO peak of the other. There is a quantitative good agreement for the range of $\Delta v$
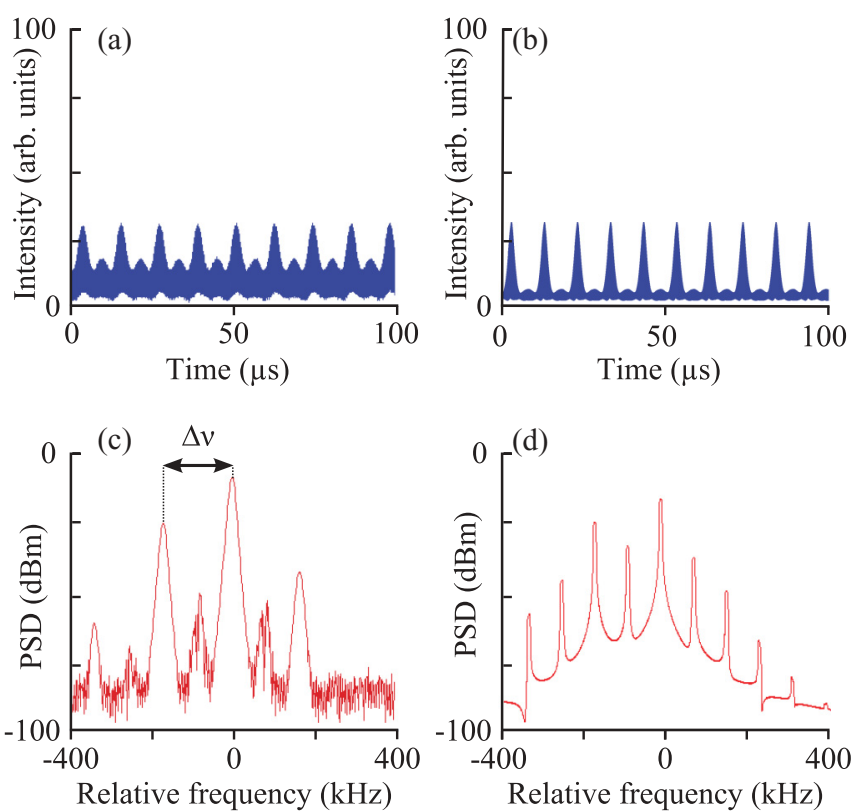

FIG. 6. (Color online) (a) Appearance of period doubling on the amplitude modulation when $\Delta v \approx 2 f_{R}$. (b) Corresponding simulated time series. (c) and (d) are the measured and simulated power spectral density, respectively.
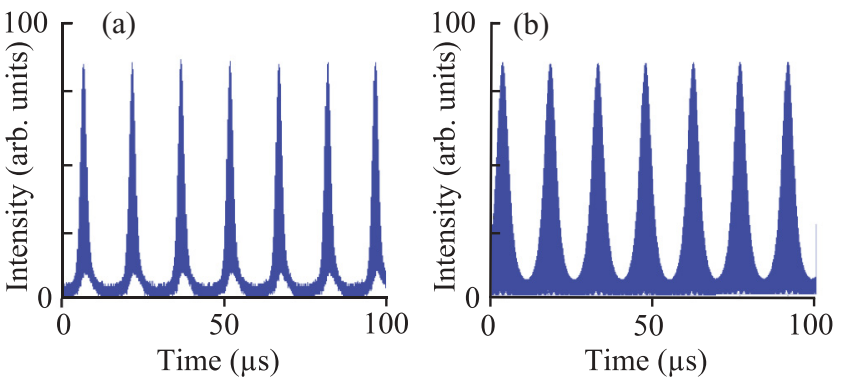

(c)
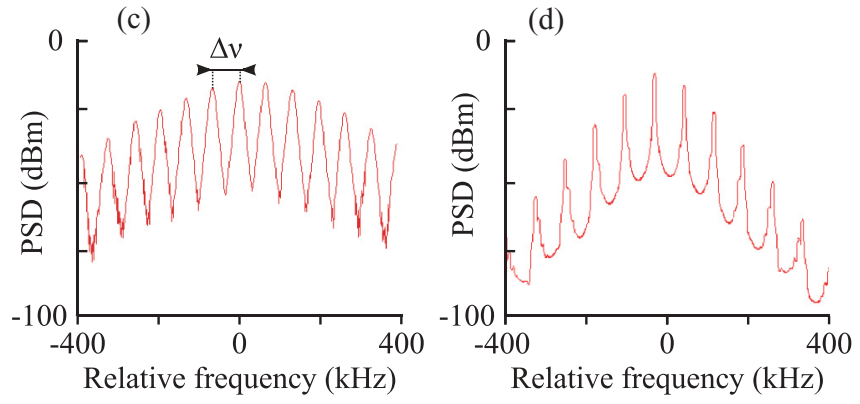

FIG. 7. (Color online) $\Delta v=1.05 f_{R}$. (a) Experimental and (b) simulated time series of $I=\left|E_{x}+E_{y}\right|^{2}$. (c) Experimental and (d) simulated power spectral density. The displayed frequencies are relative to $2 f_{A O}$.

where this regime appears. The period-doubling solution is experimentally observed when $\Delta v$ spans between $1.5 f_{R}$ and $2.5 f_{R}$, which is consistent with the numerical bifurcation diagram.

\section{Resonant modulation $\left(\Delta v \approx f_{R}\right)$}

When $\Delta v \approx f_{R}$, the resonant response of the injected mode produces a pulselike envelop of the beating intensity with a stable repetition rate equal to $\Delta v$ (Fig. 7).

\section{Low-frequency modulation and chaos $\left(f_{A}<\Delta v<f_{R}\right)$}

When $\Delta v<f_{R}$, a periodic, pulsed regime is still observed. It differs from the previous case by a low-frequency modulation of the pulses' envelop (Fig. 8), suggesting a bifurcation to quasiperiodic oscillations. The modulation becomes more and more irregular as $\Delta v$ is further reduced to approach $f_{A}$ until a chaotic signal is observed (Fig. 9).

\section{B. Phase histograms}

Our experimental setup allows us to push further the comparison between theory and experiment by measuring directly the relative phase $\Phi$. To this end, we let the $x$ and $y$ laser fields interfere on a fast photodiode after a polarizer. If $v_{y}$ is locked to the frequency $v_{x}+2 f_{A O}$, we expect that the beat signal $I(t)$ contains a fast oscillation at $2 f_{A O}$, phase locked to the second harmonic of the RF signal driving the Bragg cell. Depending on $\Delta v$, the $2 f_{A O}$ oscillation may be strongly modulated by the slow intensity fluctuations occurring over much longer time scales $\sim 1 / f_{R}$. Therefore, the measure of the phase $\Phi$ of the interference signal, relative to the phase of the second harmonic of the RF drive, gives us access to the phase of $E_{y}$, relative to the reinjected, frequency-shifted 


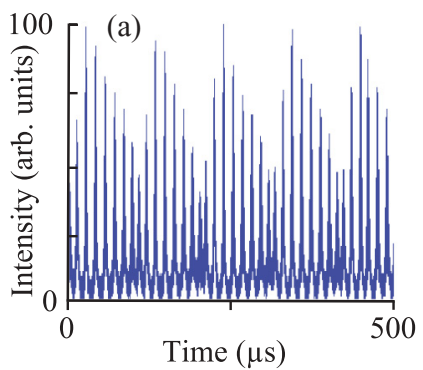

(c)
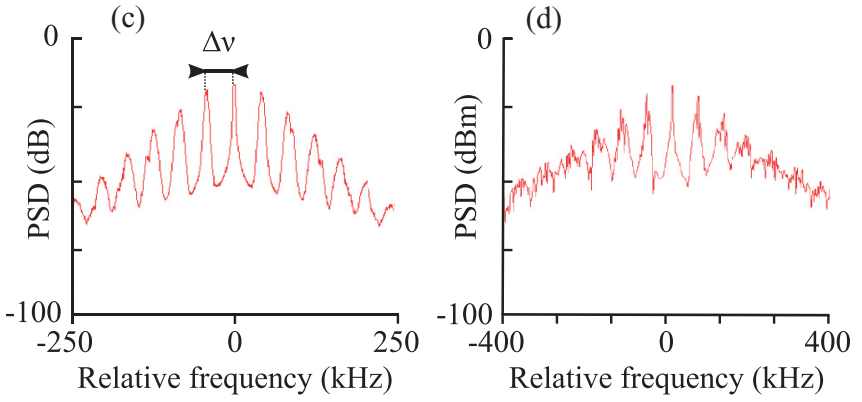

FIG. 8. (Color online) $\Delta v=0.88 f_{R}$. (a) Experimental and (b) simulated time series of $I=\left|E_{x}+E_{y}\right|^{2}$. (c) Experimental and (d) simulated power spectral density. The displayed frequencies are relative to $2 f_{A O}$.

$E_{x}$ field. Using a fast digital oscilloscope (40 GS/s), we are able to obtain histograms of the relative phase $\Phi$ between the photodiode signal and the RF drive. A single measure of the phase $\Phi$ is obtained measuring the average value of $\Phi$ over 100 periods, i.e., for a time trace of $500 \mathrm{~ns}$; each histogram contains 5000 phase measurements. The raw data
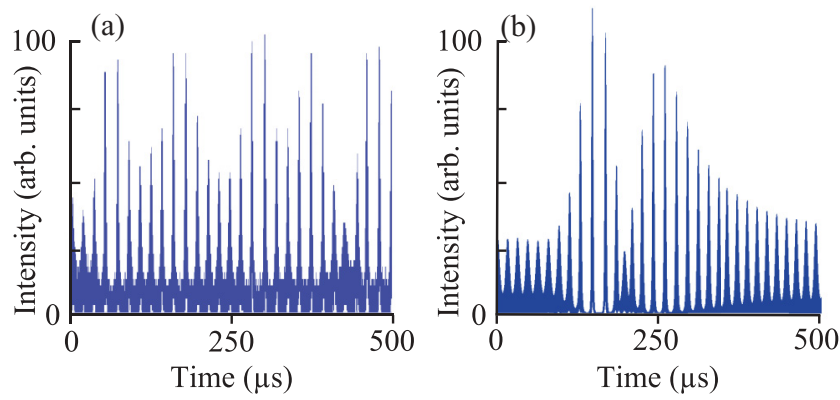

(c)
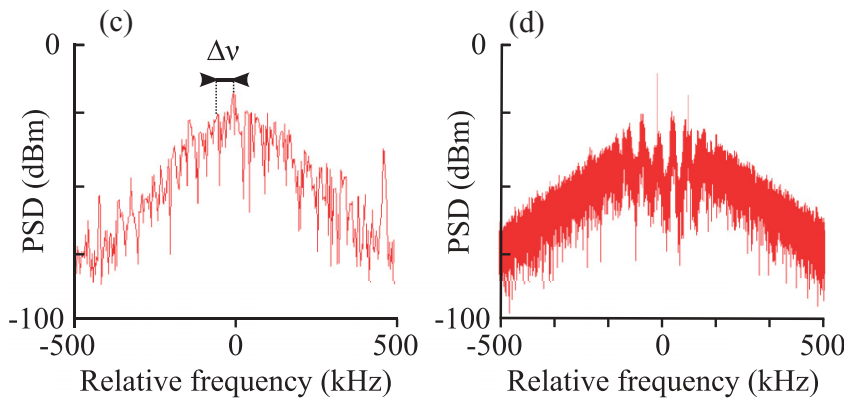

FIG. 9. (Color online) $\Delta v=0.85 f_{R}$. (a) Experimental and (b) simulated time series of $I=\left|E_{x}+E_{y}\right|^{2}$. (c) Experimental and (d) simulated power spectral density. The displayed frequencies are relative to $2 f_{A O}$.
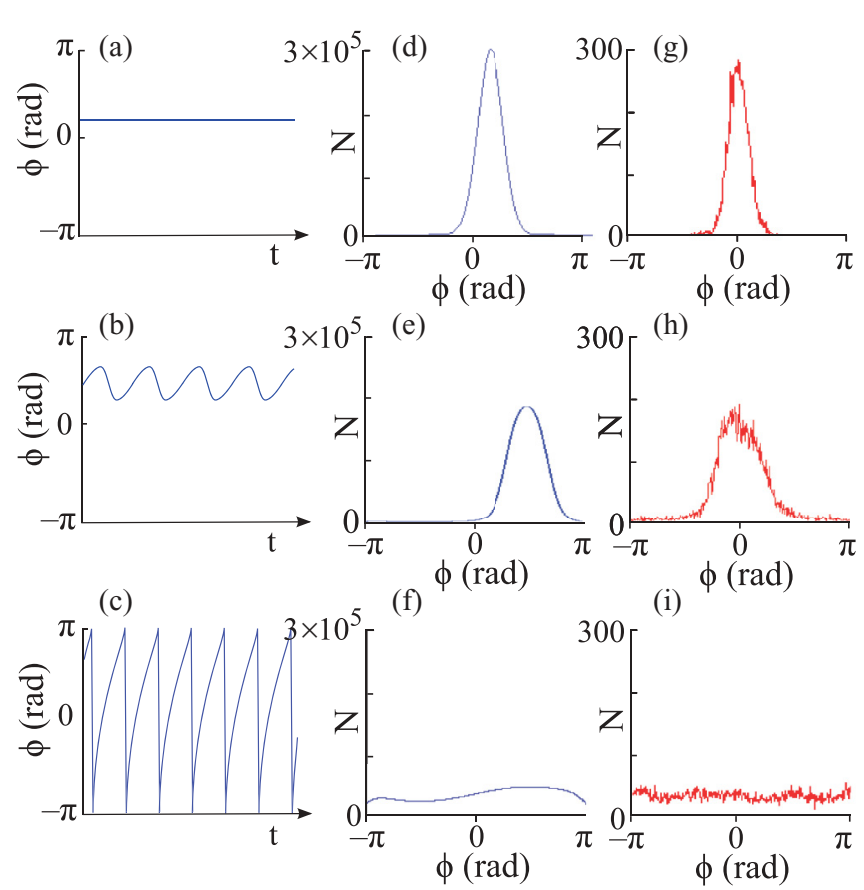

(i)

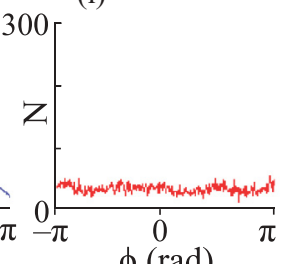

FIG. 10. (Color online) Computed time series of $\Phi$ for (a) $\Delta v=0.3 f_{A}$ (locked phase), (b) $\Delta v=1.33 f_{A}$ (bounded phase), and (c) $\Delta v=3.2 f_{A}>f_{B}$ (drifting phase). Computed histograms of $\Phi$ for the (d) locked, (e) bounded, and (f) drifting cases. (g), (h), (i) Corresponding experimental histograms. The histograms in $(\mathrm{g})$ and (h) have been centered on zero because the average value of $\Phi$ could not be measured with our setup.

are presented in Figs. 10(g), 10(h), and 10(i), together with the histograms [Figs. 10(d), 10(e), and 10(f)] obtained from the numerically calculated time series [Figs. 10(a), 10(b), and $10(\mathrm{c})]$. Figure $10(\mathrm{~g})$ shows a phase histogram when $\Delta v=0.3 f_{A}$ inside the Adler locking range. We obtain a relatively narrow peak, whose width is due to unavoidable technical imperfections. Note that the histogram from the numerical time series [Fig. 10(a)] would be a perfect Dirac delta function. In order to reproduce the experimental data, we have convoluted the histogram obtained from the deterministic (i.e., without noise terms) equations of motion with a Gaussian distribution, whose standard deviation $(\sim \pi / 12)$ is chosen in order to match the width of the experimental histogram. We stress that the measured width of the phase histogram is compatible with Fig. 4. Indeed, the measurement of a histogram takes $\sim 1 \mathrm{~s}$. A relative phase shift $\sim \pi / 12$ in $1 \mathrm{~s}$ is equivalent to a frequency drift $\sim 1 / 24 \mathrm{~Hz}$ of the beat note relative to the RF synthesizer, too small to be measured by an electrical spectrum analyzer. In order to investigate the origin of this residual phase drift, we have measured independently the differential frequency drift (i.e., the variation of $\Delta v_{0}$ over time) of the laser fields, which is equal to $0.4 \mathrm{kHz} / \mathrm{s}$. Inside the phase-locking range, a variation of $\Delta v_{0}$ produces a change in $\Phi$ through the Adler equation $\Phi=\sin ^{-1}\left(\frac{\Delta v}{f_{A}}\right)$. However, the measured value of the differential frequency drift is too small to explain entirely the width of the histogram. We, therefore, attribute the residual phase shift to a drift of the phase associated to the feedback path, which is assumed to be constant in the model. Indeed, the feedback-cavity length is 


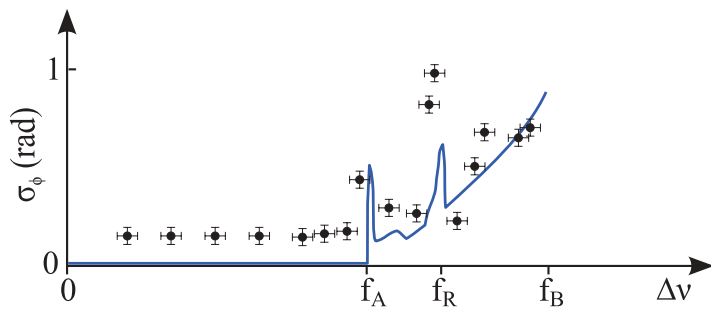

FIG. 11. (Color online) Standard deviation $\sigma_{\Phi}$ of $\Phi$ vs $\Delta v$. The lines represent the numerical integration, and the dots represent the experimental data. The standard deviations are calculated for the time series of duration $T=2.5 \mathrm{~ms}$. Note that no experimental points are marked beyond $f_{B}$ simply because there the histograms are flat, such as in Fig. 4(b).

not actively stabilized, and an optical-path variation as small as $\lambda / 48$ over $1 \mathrm{~s}$ is sufficient to produce the observed phase drift. Figures 10(e) and 10(h) show the calculated and experimental histograms for $\Delta v=1.33 f_{A}$ in the bounded-phase region. The experimental histogram is larger than that in Fig. $10(\mathrm{~g})$ but shows that the relative phase remains trapped in a bounded region of the $[-\pi, \pi]$ interval, indicating that synchronization occurs even beyond $f_{A}$. We note that, without including noise, the time series in Fig. 10(b) produces a two-peak histogram for the bounded phase. When this histogram is convoluted with the previously deduced normal distribution, a good agreement with the experimental histogram is found. Finally, for $\Delta v=3.2 f_{A}$, well outside the Adler locking range, the relative phase drift results in a flat histogram [Figs. 10(c), 10(f), and 10(i)].

In Fig. 11 we compare the measured value of the standard deviation $\sigma_{\Phi}$ of $\Phi$ to the computed value from the model as a function of $\Delta v$. The theory and the experiment show good agreement; the general evolution of the theoretical curve is well reproduced and so is the behavior around $f_{A}$ and $f_{R}$. The experimental points are obtained from histograms such as those presented in Fig. 10. The existence and the boundaries of an average frequency-synchronization range extending up to the frequency $f_{B}$ beyond the Adler phase-locking region are clearly evidenced. The experimental and numerical values of $f_{B}$ show very good agreement.

\section{Additional remarks \\ 1. Frequency $f_{B}$}

As we compare the bifurcation diagram for the intensity (Fig. 2) with the bifurcation diagram for the phase (Fig. 3), we note no changes in the intensities when $\Delta v$ is close to $f_{B}$. We can gain insight into this transition by examining the real and the imaginary parts of the field $E_{y}$ in the complex plane (Fig. 12). In Figs. 12(a), 12(c), and 12(e), we note that the trajectory of the vector $E_{y}$ can be rather complex, but it never makes a complete loop around the origin. Therefore, these three cases correspond to phase trapping. On the other hand, in Figs. 12(b), 12(d), and 12(f), the trajectory makes a loop around the origin so that the phase is unbounded. $f_{B}$ is the value of $\Delta v$ for which the trajectory connects the origin $[12,20]$. No bifurcation takes place for this value of $\Delta v$, and there is no signature of it on the intensity
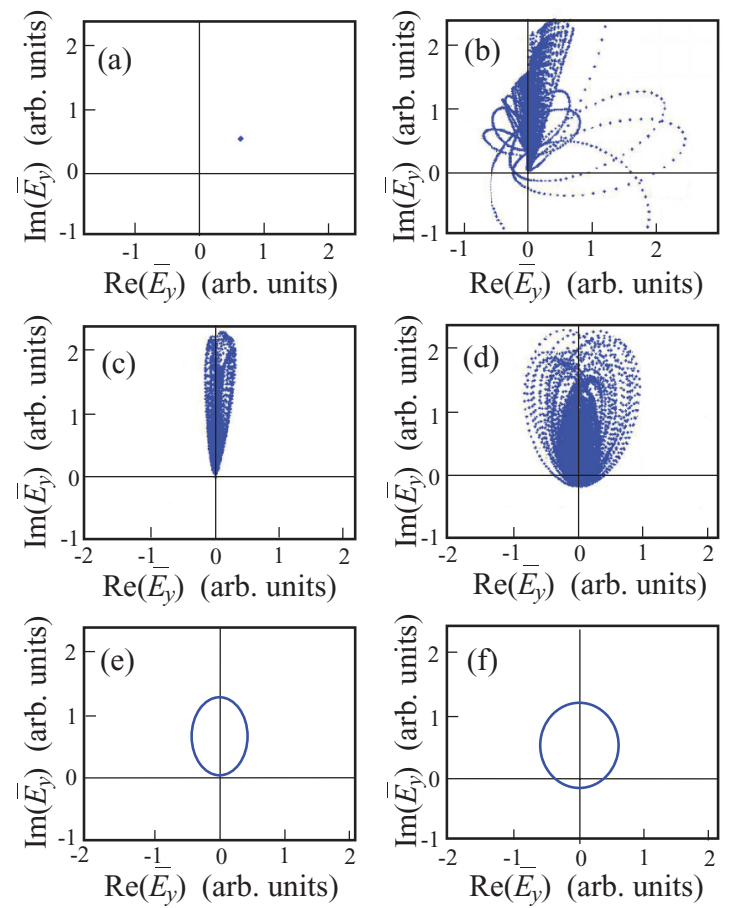

FIG. 12. (Color online) Plots of the vector $\bar{E}_{y}$ as a function of time in the planes $\operatorname{Re}\left\{\bar{E}_{y}\right\}$ and $\operatorname{Im}\left\{\bar{E}_{y}\right\}$ for different values of $\Delta v$. (a) $\Delta v=0$, (b) $\Delta v=0.81 f_{R}$, (c) $\Delta v=0.85 f_{R}$, (d) $\Delta v=f_{R}$, (e) $\Delta v=1.2 f_{R}$, and (f) $\Delta v=1.5 f_{R}$.

dynamics. These kinds of phasor plots are also accessible experimentally as was recently shown in Refs. [13,14] using an interferometric technique. In particular, these works have given clear evidence of the occurrence of boundedphase dynamics for an optically injected semiconductor laser.
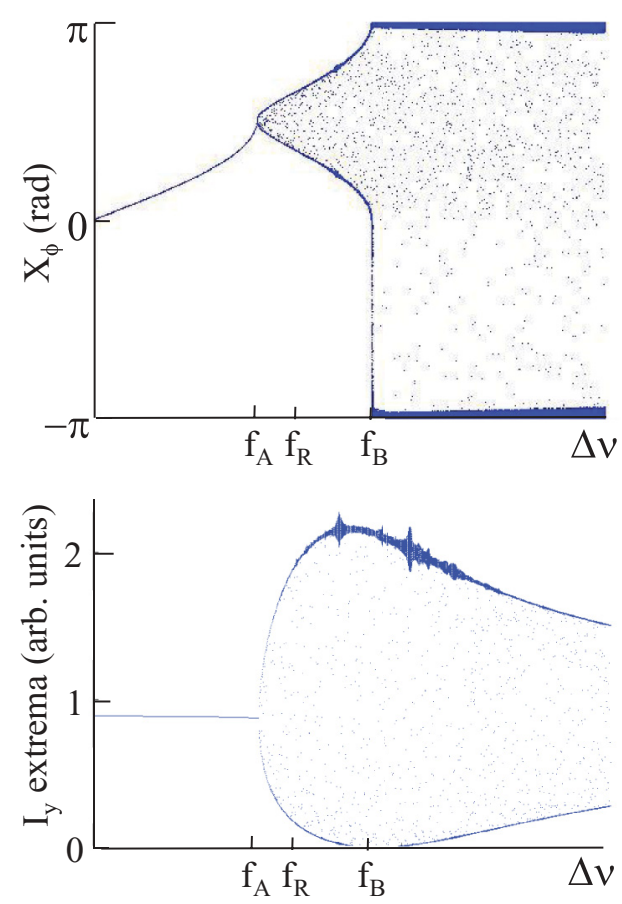

FIG. 13. (Color online) Bifurcation diagrams for $\beta=0$. 


\section{Role of $\beta$}

In our setup, the frequency-shifted $E_{x}$ field is optically injected in the $E_{y}$ field. This coherent coupling is not the only one present in the system because the two modes are also incoherently coupled via the cross saturation in the gain medium, which is accounted for by $\beta$. This raises the question on the precise role of $\beta$. In Fig. 13, we report the numerically computed bifurcation diagrams for $\beta=0$. In this case, $E_{x}$ and $n_{x}$ completely decouple from the other variables, and one is left with the important and thoroughly studied problem of a laser undergoing optical injection [20].

In this simpler case, the steady state undergoes a smooth Hopf bifurcation at the locking point $f_{A}$, and a branch of time-dependent, bounded-phase solutions appears for $f_{A}<$ $f<f_{B}$. Comparing with the bifurcation diagram in Fig. 3 , we note that $\beta$ is responsible for higher-order bifurcations near $f_{A}$ and $f_{R}$.

\section{CONCLUSION}

In this paper, we reported a systematic experimental and numerical study of the dynamics of a dual-frequency laser as the two laser modes are coherently coupled by optical frequency-shifted feedback, resonant with the laser-relaxationoscillation frequency. It extends the work in Ref. [9] by detailing the various bifurcation changes occurring through the phase-locking phenomenon. Both the intensities and the relative phase are accessible experimentally, allowing quantitative comparisons between experimental and numerical bifurcation diagrams.

A two-mode rate-equation model, supplemented with a term describing the optical feedback, has been used to interpret the experimental results. In particular, the model predicts the existence of a phase-locking range, of a frequency-locking without the phase-locking range, and their extent as well as several intensity dynamics, such as, e.g., resonant modulation, chaos, and period doubling. In our opinion, given the overall complexity of the dynamics, the agreement obtained between the theoretical predictions and the experiments is quite remarkable and validates the assumptions on which the model was built.

In the system under study, the optical feedback provides coherent coupling between the two modes. Furthermore, they are also incoherently coupled via cross saturation in the gain medium. We studied its effect numerically by comparing bifurcation diagrams with or without this incoherent coupling. We show that the latter is responsible for higher-order bifurcations leading to chaotic output in the vicinity of the phase-locking point.

We stress that, even if the resonant character of the feedback makes it easier to recognize the transition from phase-locking to bounded-phase dynamics, because of the huge effect on the intensity dynamics as soon as the detuning $\Delta v$ is bigger than the Adler locking range $f_{A}$, frequency locking without phase locking is, however, a universal kind of synchronization. For instance, it occurs in coupled van der Pol oscillators [10] and in interacting cavity solitons $[21,22]$ and does not necessarily require the presence of a resonance [14]. We plan to verify this possibility by exploring nonresonant cases in the near future.

A natural extension of the present work concerns semiconductor lasers, such as two-frequency vertical-external-cavitysurface-emitting-lasers (VECSELs) [23]. Two particular features of semiconductor lasers then need to be taken into account. First, there is an intrinsic amplitude-phase coupling, described by the Henry factor, which provides another coherent coupling mechanism. Second, semiconductor lasers have much faster intrinsic dynamics compared to diode-pumped solid-state lasers, meaning that the feedback delay can no longer be neglected [24,25].

\section{ACKNOWLEDGMENTS}

This work was partially supported by the contract "MINOTOR" in the frame of the Délégation Générale de l'Armement (DGA) program ASTRID and by the Contrat de Projet Etat-Région PONANT. M.V. thanks the Foundation of the University Rennes 1 for a research grant.
[1] Unlocking Dynamical Diversity: Optical Feedback Effects on Semiconductor Lasers, edited by D. M. Kane and K. A. Shore (Wiley and Sons, Chichester, 2005).

[2] B. Dahmani, L. Hollberg, and R. Drullinger, Opt. Lett. 12, 876 (1987).

[3] K. Otsuka and J.-L. Chern, Opt. Lett. 16, 1759 (1991).

[4] E. Lacot, R. Day, J. Pinel, and F. Stoeckel, Opt. Lett. 26, 1483 (2001).

[5] S. Blaize, B. Bérenguier, I. Stéfanon, A. Bruyant, G. Lérondel, P. Royer, O. Hugon, O. Jacquin, and E. Lacot, Opt. Express 16, 11718 (2008).

[6] L. Kervevan, H. Gilles, S. Girard, and M. Laroche, Opt. Lett. 32, 1099 (2007).

[7] M. Brunel and M. Vallet, Opt. Lett. 33, 2524 (2008).

[8] J. Thévenin, M. Vallet, M. Brunel, H. Gilles, and S. Girard, J. Opt. Soc. Am. B 28, 1104 (2011).
[9] J. Thévenin, M. Romanelli, M. Vallet, M. Brunel, and T. Erneux, Phys. Rev. Lett. 107, 104101 (2011).

[10] T. Chakraborty and R. H. Rand, Int. J. Nonlinear Mech. 23, 369 (1988).

[11] P. A. Braza and T. Erneux, Phys. Rev. A 41, 6470 (1990).

[12] M. K. Stephen Yeung and S. H. Strogatz, Phys. Rev. E 58, 4421 (1998).

[13] B. Kelleher, D. Goulding, B. Baselga-Pascual, S. P. Hegarty, and G. Huyet, Eur. Phys. J. D 58, 175 (2010).

[14] B. Kelleher, D. Goulding, B. Baselga-Pascual, S. P. Hegarty, and G. Huyet, Phys. Rev. E 85, 046212 (2012).

[15] M. Brunel, O. Emile, F. Bretenaker, A. Le Floch, B. Ferrand, and E. Molva, Opt. Rev. 4, 550 (1997). 
[16] K. Otsuka, P. Mandel, S. Bielawski, D. Derozier, and P. Glorieux, Phys. Rev. A 46, 1692 (1992).

[17] T. Erneux and P. Glorieux, Laser Dynamics (Cambridge University Press, Cambridge, 2010).

[18] M. Brunel, A. Amon, and M. Vallet, Opt. Lett. 30, 2418 (2005).

[19] M. Brunel, O. Emile, M. Vallet, F. Bretenaker, A. Le Floch, L. Fulbert, J. Marty, B. Ferrand, and E. Molva, Phys. Rev. A 60, 4052 (1999).

[20] S. Wieczorek, B. Krauskopf, T. B. Simpson, and D. Lenstra, Phys. Rep. 416, 1 (2005).
[21] H. Vahed, R. Kheradmand, H. Tajalli, G. Tissoni, L. A. Lugiato, and F. Prati, Phys. Rev. A 84, 063814 (2011).

[22] P. V. Paulau, C. McIntyre, Y. Noblet, N. Radwell, W. J. Firth, P. Colet, T. Ackemann, and G.-L. Oppo, arXiv:1112.4867v1 [physics.optics].

[23] G. Baili, L. Morvan, M. Alouini, D. Dolfi, F. Bretenaker, I. Sagnes, and A. Garnache, Opt. Lett. 34, 3421 (2009).

[24] G. Giacomelli, F. Marin, and M. Romanelli, Phys. Rev. A 67, 053809 (2003).

[25] T. Erneux, Applied Delay Differential Equations (Springer, New York, 2009). 